Benchmarks

\title{
Robust ordered mRNA differential display: an improved method for global gene expression profiling
}

\author{
Honggao Liu, Jihong Hu, Lei Pan, Shuzhen Wang, Ying He, \\ and Yi Ding \\ State Key Laboratory of Hybrid Rice, Department of Genetics, \\ College of Life Sciences, Wuhan University, Wuban, China
}

BioTechniques 51:271-275 (October 2011) doi 10.2144/000113752

Keywords: global gene expression profiling; mRNA differential display; complementary DNA amplified fragment length polymorphisms

Supplementary material for this article is available at www.BioTechniques.com/article/113752.

Global gene expression profiling (GGEP) plays a pivotal role in biological research. We developed an improved GGEP method called "robust ordered mRNA differential display (RoDD)" by combining mRNA differential display (DD) and complementary DNA amplified fragment length polymorphisms (cDNA-AFLP) using elaborately designed primers and a poly(dT:A) replacement technique. Redundancy was minimized by bead-based isolation and coverage was improved by using restriction enzymes that recognized 4-bp sites. This method offers the common virtues of gel-based methods along with the reliability of cDNA-AFLP. The most significant advantage of RoDD over current gelbased methods is greatly improved coverage and minimized redundancy.

Global gene expression profiling (GGEP) provides a valuable tool for investigating genes and transcripts on a genome-wide scale. Principally, GGEP strategies fall into three categories: (i)sequencingbased, (ii) hybridization-based, and (iii) gel-based (1). Although global sequencing and microarrays are stateof-the-art, gel-based strategies remain useful for their simplicity, straightforwardness, and universality $(2,3)$.

mRNA differential display (DD) is a traditional gel-based method that amplifies single-stranded cDNA using the HindIII-T $\mathrm{T}_{11} \mathrm{~V}$ (V is $\mathrm{A}, \mathrm{G}$ or $\mathrm{C}$ ) and HindIII-7-nt-arbitrary primers (4). It is polymorphic in anchoring the $3^{\prime}$-untranslated region (5). However, the primers are so short that DD suffers effects of low stringency PCR conditions including non-specific amplifications and false-positive results; additionally, the arbitrary-7-nt-dependent primer leads to high redundancy (6). Comple- mentary DNA amplified fragment length polymorphisms (cDNA-AFLP) amplifies cDNA restriction fragments using adapter-specific primers and minimizes redundancy by a bead-based isolation technique (7). Nevertheless, it is limited by insufficient coverage since it depends on the presence of both $(6 / 4$ bp) restriction sites (8).

The reliability of DD methods may be enhanced by altering primers (9-12), while coverage of CDNA-AFLP may be optimized by varying restriction enzyme numbers and frequency of cutting $(8,13,14)$. One notable success was achieved by introducing

$$
\left.\operatorname{BstwIY(} \mathrm{Y}_{\mathrm{Y}{ }_{\mathrm{CTAG}} \mathrm{GATC}}^{\mathrm{R}}, \mathrm{R} \text { is } \mathrm{A} \text { or } \mathrm{G}, \text { and } \mathrm{Y} \text { is } \mathrm{T} \text { or } \mathrm{C}\right)
$$

or

$$
\operatorname{ApoI}\left(\begin{array}{c}
\mathrm{R}^{\vee} \\
\mathrm{Y}^{\mathrm{AATT}} \mathrm{Y} \\
\text { TTAA }_{\wedge} \mathrm{R}
\end{array}\right)
$$

to the bead-based cDNA-AFLP protocol, (14) but this technique has not been widely applied.

Here we report an improved GGEP method that combines DD and cDNA-AFLP (Figure 1). The procedure begins by introducing a $5^{\prime}$-biotin[protection bases]-[Acul site]-3' sequence $16 \mathrm{bp}$ upstream of the poly(dT:A) boundary of the cDNA, followed by detecting length polymorphisms of the 3 -restriction fragments using a bead-based cDNA-AFLP protocol that relies on

$$
A c u\left[\left[\begin{array}{l}
\text { CTGAAG }(\mathrm{N})_{16}^{\vee} \\
\text { GACTTC }(\mathrm{N})_{14}
\end{array}\right]\right.
$$

and a frequent cutter such as $M s p I$ $\left(\mathrm{C}^{\vee} \mathrm{CGG}\right)$. This method offers the reliability of cDNA-AFLP using adapterspecific primers and high stringency PCR conditions along with the polymorphic nature of DD through anchoring of the 3 '-untranslated region. Because the artificial AcuI sites allow full coverage of $3^{\prime}$ ends of mRNAs, the final coverage is dependent on the orderly presence of a 4 -bp restriction site in the $5^{\prime}$ ends. The biotin aids in minimizing redundancy through streptavidin-coated magnetic beads. The MlyI site

$$
\left[\begin{array}{l}
\mathrm{GAGTC}(\mathrm{N})_{5}^{\vee} \\
\mathrm{CTCAG}(\mathrm{N})_{5}
\end{array}\right]
$$

was designed as protection bases to help eliminate the artificial heels when necessary. This method was named "robust ordered mRNA differential display" (RoDD).

A validation experiment was performed. Total RNA was isolated from the leaves and roots of one-week-old rice seedlings (Oryza sativa L.) with TRIzol Reagent (Life Technologies, Carlsbad, CA, USA), followed by purification and quantity and quality assessment as described (4). Single-stranded cDNA was synthesized with PrimerScript Reverse Transcriptase (Takara, Dalian, China) using $500 \mathrm{ng}$ total RNA and 100 pmol 5'-biotin-MlyI-AcuI-T $-\mathrm{V}$ primers $\left(b^{2} \mathrm{~T}_{16} \mathrm{~V}, 5^{\prime}\right.$-biotin-GAGTC $\mathrm{T}$ GAAGTTTTTTTTTTTTTTTTV-3'; $\mathrm{V}$ are anchoring bases composed of $\mathrm{A}$, $G$ and $C)$. Double-stranded cDNA was synthesized by nick translation using RNase H, DNA polymerase I, and Escherichia coli ligase (Takara) and purified using AxyPrep PCR Clean-up kit (Axygen, Union City, CA, USA), followed by digestion using $10 \mathrm{U} M s p \mathrm{I}$ (New England Biolabs, Ipswich, MA, 
USA) and ligation to $50 \mathrm{pmol} M s p \mathrm{I}$ adapter (Table 1) using 200 U T4 ligase (New England Biolabs). The biotinylated fragments were trapped by $50 \mu \mathrm{L}$ activated streptavidin-coated magnetic beads (Promega, Madison, WI, USA) in a magnetic field in $1 \times$ STEX buffer (1.0M NaCl, 10.0mM Tris-HCl pH 8.0, $1.0 \mathrm{mM}$ EDTA, 1\% Triton X-100) and washed with $1 \times$ STEX buffer to remove the redundant fragments, followed by digestion using $5 \mathrm{U} A c u \mathrm{I}$ (New England Biolabs) to release the target fragments from the bead-immobilized biotinylated ends, with a $5^{\prime}$-AA or $5^{\prime}$-NN dinucleotide overhang retained. The $5^{\prime}-\mathrm{NN}$ overhangs were derived from the existing AcuI sites in some restriction fragments. The released fragments (in supernatant) were divided into two aliquots and marked PTT and PNN, followed by ligation to 50 pmol PTT adapter and PNN adapters ( $\mathrm{N}$ is the mixture of $\mathrm{A}, \mathrm{G}, \mathrm{C}$ and T; Table 1) using $100 \mathrm{U}$ T4 ligase. The solution was diluted 5 -fold with distilled water and used as the templates for pre-amplification with the primer combination of $\mathrm{P}+0 / \mathrm{M}+0$, followed by selective amplifications as described (7). The selective primer combinations for the PTT and $\mathrm{PNN}$ templates are $\mathrm{P}+\mathrm{VN} / \mathrm{M}+\mathrm{NN}$ and $\mathrm{P}+\mathrm{NN}$ (excluding $\mathrm{P}+\mathrm{TT}$ )/ $\mathrm{M}+\mathrm{NN}$ (Table 1), respectively. Amplified fragments were separated by $4 \%$ denaturing PAGE, visualized by silver staining (15), and recorded with a charge-coupled device (CCD) camera. The band patterns were analyzed with BandScan 4.30 software (Glyko, Novato, CA, USA).

The results showed that RoDD has high coverage and good reliability. There were 2128 and 1628 transcript-derived fragments (TDFs) obtained using 32 PTT and 30 PNN primer combinations, respectively (Figure 2). Assuming that 192 PTT $(12 \times 16=192)$ and 240 PNN $[(16-1) \times 16=240)]$ primer combinations were completely used, $1.28 \times$ $10^{4}$ and $1.30 \times 10^{4}$ transcripts could be covered, respectively, summing up to $2.58 \times 10^{4}$. There were $920(31.46 \%)$ and 832 (29.34\%) differentially expressed TDFs [DE-TDFs] detected in the leaves and roots, respectively. Forty DE-TDFs were randomly picked, recovered, cloned and sequenced as described (15). Thirtynine DE-TDFs were mapped on rice cDNAs using the NCBI Blast Network service (http://www.ncbi.nlm.nih.gov/ BLAST/) (Supplementary Table S1) and 34 DE-TDFs were confirmed to be true positives (Supplementary Figure S1) by a semiquantitative $\mathrm{RT}$-PCR validation method (16).

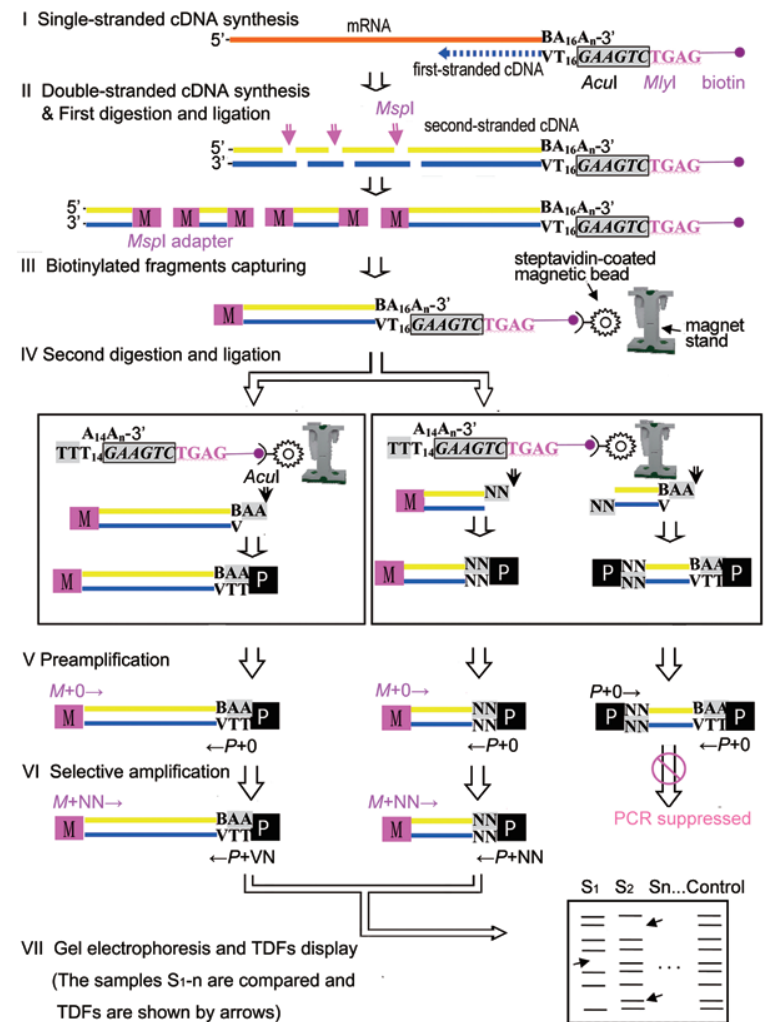

Figure 1. The work flow of the RoDD method. Double-stranded cDNA was synthesized routinely using total RNA and bmaT ${ }_{16} V$ primers to introduce a 5'-biotin-Mlyl-Acul- ${ }_{16}$ sequence upstream of the poly(dT:A) boundary. This was digested by $\mathrm{Mspl}$ and ligated to the $\mathrm{Mspl}$ adapter. Biotinylated fragments were trapped by streptavidin-coated magnetic beads to minimize redundancy, followed by digestion using the asymmetrically cutting enzyme, Acul, to release the target fragments (in supernatant) from the bead-immobilized biotinylated ends, with a 5'-AA or 5'-NN dinucleotide overhang retained. The supernatant was divided into two aliquots and linked to the PTT adapter or PNN adapters. The ligation products served as templates for pre-amplification using the primer combination of $\mathrm{P}+\mathrm{O} / \mathrm{M}+\mathrm{O}$, followed by selective amplifications using selective primer combinations $[\mathrm{P}+\mathrm{VN} / \mathrm{M}+\mathrm{NN}$ and $\mathrm{P}+\mathrm{NN}($ excluding $\mathrm{P}+\mathrm{TT}) / \mathrm{M}+\mathrm{NN}$ for PTT and PNN templates]. The amplicons were separated in a $4 \%$ polyacrylamide gel, followed by silver staining and subsequent analysis.

Table 1. Primers and adapters involved in the RoDD method

Name Sequence $\left(5^{\prime}\right.$ to $\left.3^{\prime}\right) \quad$ Purpose

\begin{tabular}{|c|c|c|}
\hline bmaT $_{16} \mathrm{~V}$ & biotin-GAGTCTGAAGTTTTTTTTTTTTTTTTTV & Mixture; for RT-PCR \\
\hline Mspl adapter & $\begin{array}{l}\text { M-F: GATGATGAGTCCTGAA } \\
\text { M-R: } \underline{\text { CGTTCAGGACTCAT }}\end{array}$ & PCR template preparation \\
\hline PTT adapter & $\begin{array}{l}\text { PTT-F: CTCGTAGACTGCGAACCTT } \\
\text { PTT-R: GGTTCGCAGTC }\end{array}$ & PCR template preparation \\
\hline PNN adapters & $\begin{array}{l}\text { PNN-F mixture: CTCGTAGACTGCGAAC- } \\
\text { CTTNN } \\
\text { PNN-R: AAGGTTCGCAGTC }\end{array}$ & $\begin{array}{l}\text { Mixture; for PCR template prepa- } \\
\text { ration }\end{array}$ \\
\hline $\mathrm{P}+0$ & CTCGTAGACTGCGAACCTT & Pre-amplification \\
\hline $\mathrm{M}+0$ & GATGATGAGTCCTGAACGG & Pre-amplification \\
\hline $\mathrm{P}+\mathrm{VN}$ & GTAGACTGCGAACCTTVN & $\begin{array}{l}\text { Selective } \\
\text { amplification }\end{array}$ \\
\hline $\mathrm{P}+\mathrm{NN}$ & GTAGACTGCGAACCTTNN & $\begin{array}{l}\text { Selective } \\
\text { amplification }\end{array}$ \\
\hline $\mathrm{M}+\mathrm{NN}$ & GATGAGTCCTGAACGGNN & $\begin{array}{l}\text { Selective } \\
\text { amplification }\end{array}$ \\
\hline
\end{tabular}

bmaT $V$ represents $5^{\prime}$-biotin-Mlyl-Acul-T $\quad-\mathrm{V}-3^{\prime}$. Acul is an asymmetrically cutting enzyme with a cleavage site 16 bases downstream of its recognition site. The adapters and primers are divided into two series, $M$ $(M s p l)$ and $\mathrm{P}($ Acul $) . V$ represents $\mathrm{A}, \mathrm{G}$, and/or $\mathrm{C}$, and $\mathrm{N}$ represents $\mathrm{A}, \mathrm{G}, \mathrm{C}$, and/or $\mathrm{T}$. The protruding ends of the adapters are underlined. The NN in the P+NN excludes TT. 
A

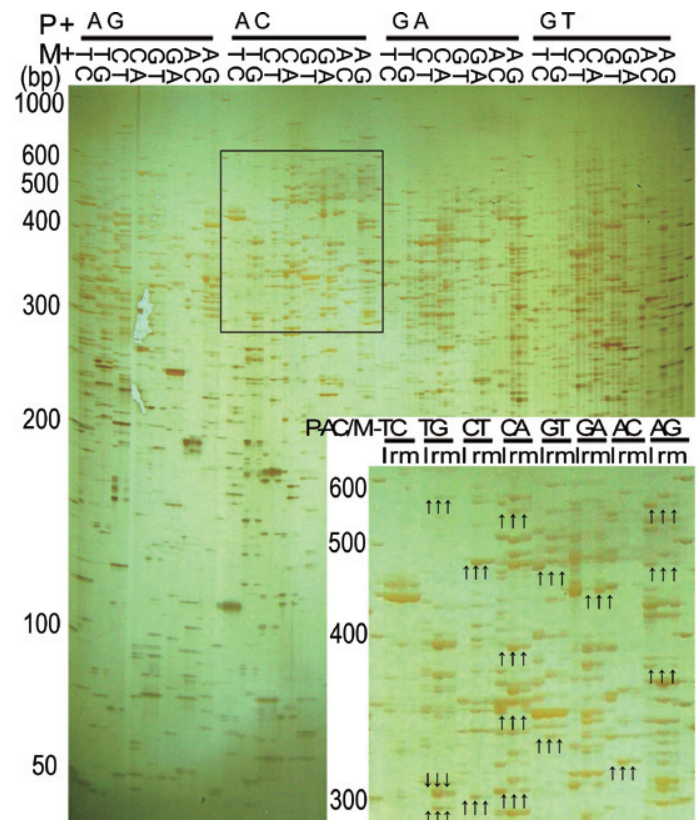

B

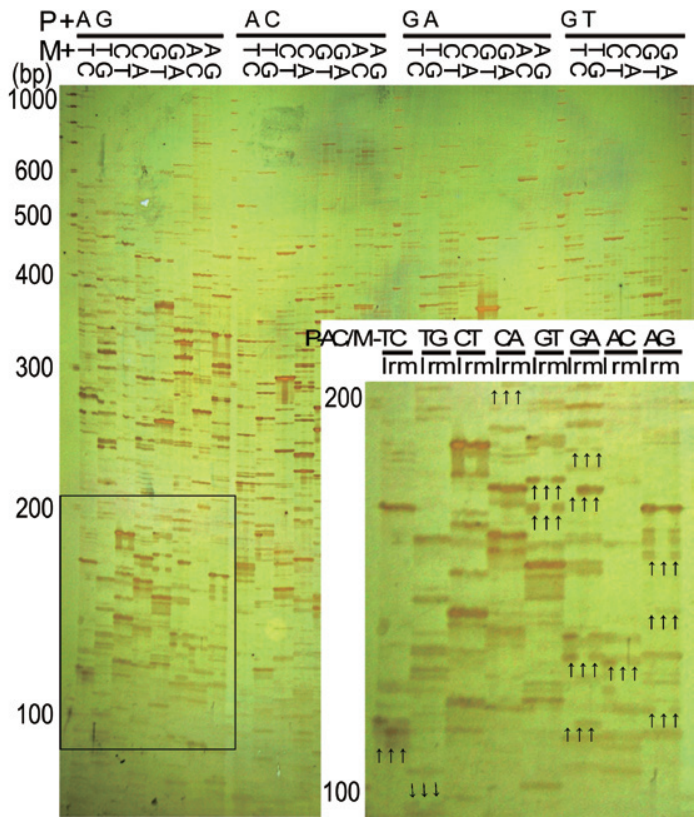

Figure 2. RoDD patterns of rice leaves and roots. The RoDD pattern derived from PCRs using 32 and 30 primer combinations for the PTT (A) and the PNN (B) templates, respectively. Double-scaled views of the local parts (in black box) are presented for each illustration and some DE-TDFs are shown by arrows. P refers to the pseudo primer (for PTT adapter or PNN adapters) and M to the Mspl primer. There were three lanes per primer combination. The I, $r$ and $\mathrm{m}$ refer to rice leaves, roots and their mixture (internal control), respectively. The marker is 100bp DNA ladder (TransGen Biotech, Beijing, China).

Table 2. Coverage prediction of the RoDD method by mathematical modelling and an in silico assay

\begin{tabular}{|c|c|c|c|c|c|c|c|}
\hline Species & Total & With No Mspl site & $<14$ bp & $>1000$ bp & Valid bands & \multicolumn{2}{|c|}{ Coverage (\%) } \\
\hline & & & & & & In silico & Mathematical \\
\hline Rice & 67392 & 5324 & 2493 & 2585 & 56990 & 84.56 & 92.75 \\
\hline Human & 29159 & 2722 & 782 & 1707 & 23948 & 82.13 & 92.79 \\
\hline
\end{tabular}

The mathematical model was established based on the binomial probability density function, and the in silico assay was performed with a site coverage analysis program written in Perl. The published transcripts of human (ftp://ftp1.nci.nih.gov/pub/MGC/fasta/) and rice (ftp://ftp.plantbiology.msu.edu/pub/data/BFGR/model_genome_sequences/) were used. The transcripts with final TDF sizes of 14-1000 bp (50-1036 bp including the adapters) were regarded as validly covered due to the resolution of polyacrylamide gels and the minimum informative sequence length in accordance with the standard of the serial analysis of gene expression.

A miniature assay confirmed the reliability of RoDD (Supplementary Figure S2); another validation assay indicated that RoDD is applicable to rice, Arabidopsis thaliana, and mouse, and that RoDD has many advantages over closely related methods (Supple- mentary Figure S3). A draft mathematical model (Supplementary Figure S4) and an in silico simulation assay revealed that RoDD has $>80 \%$ coverage on human and rice transcripts (Table 2), which is higher than currently used gel-based methods (Supplementary Table S2).

\section{Future of BIOTECH OPTICS.}

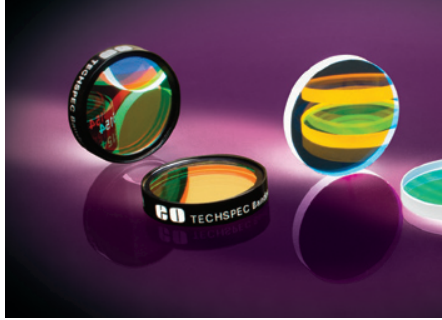

- Precision Filters, Aspheres, Objectives and more

- Over 24,200 Optics Available in Volume

- Request your FREE catalog!

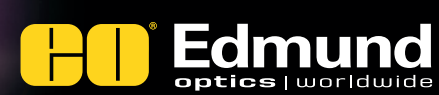

856.547.3488 | www.edmundoptics.com
The RoDD method has many advantages, including simplicity, straightforwardness, universality, reliability, high coverage and low redundancy. Furthermore, there is room for enhancement: the LI-COR DNA sequencers and infrared dye detection technology can improve accuracy and sensitivity and enable automation; many frequent cutters are available to increase coverage; and the assay may be modified into a high-throughput sequencing version. Thus, this method holds promise for global gene expression profiling in a variety of cells and tissues.

\section{Acknowledgements}

This work was supported by the National Natural Science Foundation of China (30971740, 30821064), and the National Basic Research Program (973) of China (2007CB108705). 


\section{Competing interests}

The authors declare no competing interests.

\section{References}

1. Shimkets, R.A., D.G. Lowe, J.T.-N. Tai, P. Sehl, H. Jin, R. Yang, P.F. Predki, B.E.G. Rothberg, et al. 1999. Gene expression analysis by transcript profiling coupled to a gene database query. Nat. Biotechnol. 17:798-803.

2. Korpelainen, H. and K. Kostamo. 2010. An improved and cost-effective cDNA-AFLP method to investigate transcription-derived products when high throughput sequencing is not available. J. Biotechnol. 145:43-46.

3. Sestili, S., A. Polverari, L. Luongo, A. Ferrarini, M. Scotton, J. Hussain, M. Delledonne, N. Ficcadenti, and A. Belisario. 2011. Distinct colonization patterns and cDNA-AFLP transcriptome profiles in compatible and incompatible interactions between melon and different races of Fusarium oxysporum f. sp. melonis. BMC Genomics 12:122.

4. Liang, P., J.D. Meade, and A.B. Pardee. 2007 A protocol for differential display of mRNA expression using either fluorescent or radioactive labeling. Nat. Protocols 2:457-470.

5. Eveland, A.L., D.R. McCarty, and K.E. Koch. 2008. Transcript profiling by $3^{\prime}$-untranslated region sequencing resolves expression of gene families. Plant Physiol. 146:32-44.

6. Liang, P. 2002. A decade of differential display. BioTechniques 33:338-346.
7. Vuylsteke, M., J.D. Peleman, and M.J.van Eijk. 2007. AFLP-based transcript profiling (cDNAAFLP) for genome-wide expression analysis. Nat. Protocols 2:1399-1413.

8. Stölting, K.N., G. Gort, C. Wüst, and A.B. Wilson. 2009. Eukaryotic transcriptomics in silico: optimizing cDNA-AFLP efficiency. BMC Genomics 10:565.

9. Matz, M., N. Usman, D. Shagin, E. Bogdanova, and S. Lukyanov. 1997. Ordered differential display: a simple method for systematic comparison of gene expression profiles. Nucleic Acids Res. 25:2541-2542.

10. Fischer, A. 2001. Restriction-mediated differential display (RMDD). P. 25B.4.1-17. In: F.M. Ausubel, R. Brent, R.E. Kingston, D.D. Moore, J.G. Seidman, J.A. Smith, K. Struhl (Eds.), Current Protocols in Molecular Biology. John Wiley \& Sons, Inc., New York, NY.

11. Lo, D., B. Hilbush, and J.G. Sutcliffe. 2001. TOGA analysis of gene expression to accelerate target development. Eur. J. Pharm. Sci. 14:191-196.

12. Kornmann, N., N. Preitner, D. Rifat, F.F. Olela, and U. Schibler. 2001. Analysis of circadian liver gene expression by ADDER, a highly sensitive method for the display of differentially expressed mRNAs. Nucleic Acids Res. 29:e51.

13. Fukumura, R., H. Takahashi, T. Saito, Y. Tsutsumi, A. Fujimori, S. Sato, K. Tatsumi, R. Araki, and M. Abe. 2003. A sensitive transcriptome analysis method that can detect unknown transcripts. Nucleic Acids Res. 31:e94.
14. Weiberg, A., D. Pöhler, B. Morgenstern, and P. Karlovsky. 2008. Improved coverage of cDNA-AFLP by sequential digestion of immobilized cDNA. BMC Genomics 9:480.

15. Chalhoub, B., S. Thibault, V. Laucou, C. Rameau, H. Höfte, and R. Cousin. 1997. Silver staining and recovery of AFLP amplification products on large denaturing polyacrylamide gels. BioTechniques 22:216-220.

16. Meadus, W.J. 2003. A semi-quantitative RT-PCR method to measure the in vivo effect of dietary conjugated linoleic acid on porcine muscle PPAR gene expression. Biol. Proced. Online 5:20-28.

Received 25 February 2011; accepted 9 September 2011.

Address correspondence: Yi Ding, State Key Laboratory of Hybrid Rice, Department of Genetics, College of Life Sciences, Wuhan University, Wuhan, China.E-mail:yiding@whu. edu.cn

To purchase reprints of this article, contact: biotechniques@fosterprinting.com

\section{A NEW STANDARD IN RNA ISOLATION \\ THE HIGHEST YIELD AND PURITY}

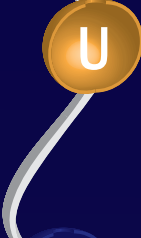

c RNAzo ${ }^{\circledR}{ }^{R} T^{*}$ isolates total RNA, with mRNA and small RNA (200-10 bases) in separate fraction.

- The single-step method without phase separation

- No DNase treatment necessary

- RNA is ready for RT-PCR, microarrays and other applications

- No need for refirigerated centrifuge

- One reagent for solid and liquid samples
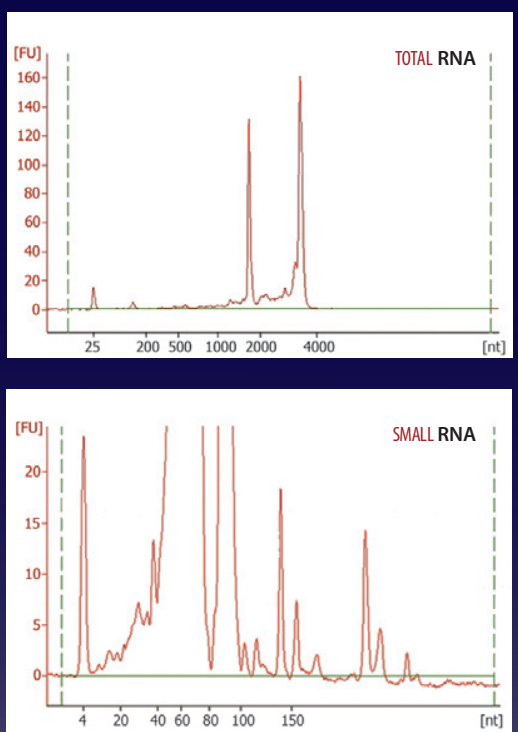